\section{Protease uninhibited}

\section{Douglas D. Richman}

THE intense media coverage of AIDS puts the public on an emotional roller coaster: inflated promises are engendered by each success and predictions of hopeless failure with each setback. The paper by Condra $e t$ al. on page 569 of this issue ${ }^{1}$, in which the authors describe the emergence of HIV-1 variants that are resistant to several protease-inhibitor drugs after treatment with just one, is likely to be interpreted as a big 'down'. The findings do indeed come as a setback, but the implications are not as gloomy as they might seem.

The drug used by Condra et al. was MK-639, which inhibits the protease activity of HIV. Just three months ago, two studies with MK-639 and ABT-538, a drug with similar antiviral characteristics, generated important observations about the population dynamics of both HIV and CD4 lymphocytes in the circulation, and about the efficacy of antiviral therapy ${ }^{2,3}$. Progress and encouragement came from the realization that high rates of destruction of CD4 lymphocytes are matched by similarly high rates of production of uninfected cells; that the high rates of production of HIV are accompanied by rapid rates of virus clearance; and that potent inhibitors of viral protease activity can rapidly reduce virus replication by as much as 1,000 times and restore CD4 cell counts, even in patients with advanced disease.

\section{Resistant virus}

Now for the setback. Condra et al. report that within 6-12 months this antiretroviral activity is largely dissipated in conjunction with the acquisition of highly resistant virus. Although disappointing, many aspects of these observations are not unexpected. On the one hand, highly resistant mutants of HIV appear to emerge with difficulty following exposure to zalcitabine (ddC), didanosine (ddI) or stavudine (d4T), dideoxynucleosides with sugars that resemble their physiological counterparts $^{4-6}$. On the other, high-level resistance readily develops with other nucleosides (zidovudine (AZT) and lamivudine (3TC)), non-nucleoside reversetranscriptase inhibitors and protease inhibitors ${ }^{7}$.

The mutability of the HIV protease is remarkable. Some 20 of the 99 amino acids in each polypeptide of this homodimeric enzyme have been shown to undergo mutation when faced with the selective pressure of various inhibitors ${ }^{8,9}$. Condra et al. now find that the cumulative acquisition of six or more of these mutations is possible, resulting both in 1,000 fold reductions in viral drug-susceptibility and in broad cross-resistance to other inhibitors against which cross-resistance is not observed with fewer mutations. As the authors point out, X-ray crystallographic models of the enzyme locate many of the mutations in the protease pocket which binds the inhibitor, thus reducing its binding affinity ${ }^{10-12}$. Other mutations at distant residues must result in conformational changes that either contribute to impaired binding or compensate for deleterious effects of drug-resistance mutations on enzyme function.

The cumulative acquisition of mutations over time within a patient, and the different evolutionary pathways that the virus takes in different patients, should not be surprising. Progressively greater resistance requires the accumulation of additive or synergistic mutations. In accommodating these mutations, the enzyme cannot tolerate compromises in its function. Such compromises would attenuate the replicative capacity of the virus. Coffin ${ }^{13}$ has argued clearly and cogently that small impairments in viral fitness would result in evolutionary failure during an infection that is characterized by high levels of virus with rapid rates of turnover. Thus many mutations compensate for impaired virus replication rather than contribute to the drug-resistance phenotype ${ }^{11}$. The different combinations of mutations that emerge may result partly from chance and partly from the baseline sequence of the virus before drug treatment. Several investigators have presented data, not yet published, that the amino-acid sequence of different isolates before treatment affects the phenotypic effects of different drug-resistance mutations.

What is the clinical significance of these observations? Unfortunately Condra et al. do not provide any information regarding the clinical status of their patients, or the viral responses (and loss thereof) with treatment. Nevertheless, the message the authors seem to convey is that monotherapy with MK-639 has no future: it abrogates the potential benefit of treatment with alternative protease inhibitors, and "multiple protease inhibitors may not prevent loss of antiviral activity resulting from resistance selection". This seemingly hopeless message, based upon four patients, is open to question. Not least, it has not stopped Merck, for whom most of the group work, from continuing to develop MK-639. It should not stop others.

There are at least three theoretical mechanisms by which antiretroviral drugs can sustain activity in the face of drug-resistant virus. First, drugs can continue to exert antiretroviral activity if plasma concentrations can be maintained that exceed the susceptibility of drugresistant virus (assuming, of course, that there are constraints on the mutability of the target protein). This approach appears to apply to some patients in whom high levels of the non-nucleoside reversetranscriptase inhibitor, nevirapine, can be achieved $^{14}$. Second, drug-resistance mutations, which confer a clear selective advantage in the face of drug pressure, may still impair the replicative capacity of the virus compared to that of the wild-type virus in the absence of treatment. Such attenuated virus may contribute to the activity of lamivudine and perhaps of multiply resistant protease mutants.

\section{Convergent therapy}

Third, when two drugs are targeted to the same viral protein (convergent therapy), mutations induced by drug 1 may sensitize the virus to drug 2 , or may prevent the emergence of viable mutants to drug 2. The mutation from methionine to valine at residue 184 of reverse transcriptase, which emerges with lamivudine treatment, suppresses the critical mutation at residue 215 that confers resistance to AZT (ref. 15). Combinations of protease inhibitors may also exploit this strategy. It seems logical that a potent inhibitor can be designed to bind to the active site of the multiply resistant viruses described by Condra et al.

Recent insights about the targets for antiviral drugs and the pathogenesis of HIV infection provide a more rational basis for developing effective treatments. Given that some people who have had long-term HIV infection have not developed AIDS, we know that a significant but incomplete suppression of virus replication is a reasonable initial aim which, if achieved, should allow patients a greater time free of disease. Setbacks are to be expected during the trek towards this goal - but they should not discourage the endeavour.

Douglas D. Richman is in the Departments of Pathology and Medicine, University of California San Diego, La Jolla, California 92093, USA.

\footnotetext{
1. Condra, J. H. et al. Nature 374, 569-571 (1995)

2. Wei, X. etal. Nature 373, 117-122 (1995).

3. Ho, D. D. et al. Nature 373, 123-126 (1995)

4. Husson, R. N Shirasaka, T. Butler K. M. Pizzo, P. A. \& Mitsuya, H.J. Pediatr. 123, 9-16 (1993).

5. Richman, D. D. etal. J. AIDS 7, 135-138 (1994)

6. Lin, P.-F. et al. J. infect. Dis. 170, 1157-1164 (1994)

7. Richman, D. D. in Textbook of AIDS Medicine (eds Broder, S., Merigan, T. C. \& Bolognesi, D.) 795-805 (Williams \& Wilkins, Baltimore, 1994)

8. Pillay, D., Bryant, M., Getman, D. \& Richman, D. D. Rev med. Virol. (in the press).

9. Mellors, J W Larder B. A. \& Schinazi, R. F. Int Antivir News 3, 8-13(1995).

10. Navia, M. A. et al. Nature 337,615-620 (1989).

11. Ho, D. D.etal.J. Virol. 68, 2016-2020 (1994).

12. Wlodawer, A. \& Erickson, J.W. A. Rev. Biochem. 62 543-585 (1993).

13. Coffin, J.M. Science 267, 483-489 (1995)

14. Havlir, D. et al.J. infect. Dis. 171, 537-545 (1995)

15. Tisdale, M., Kemp, S. D., Parry, N. R. \& Larder, B. A. Proc natn. Acad. Sci.U.S.A. 90, 5653-5656 (1993).
} 